\title{
Investigating the sub-regions of the superior parietal cortex using functional magnetic resonance imaging connectivity
}

\author{
Adnan A. S. Alahmadi (i)
}

\begin{abstract}
Objectives: Traditionally, the superior parietal lobule (SPL) is usually investigated as one region of interest, particularly in functional magnetic resonance imaging (fMRI) studies. However, cytoarchitectonic analysis has shown that the SPL has a complex, heterogeneous topology that comprises more than seven sub-regions. Since previous studies have shown how the SPL is significantly involved in different neurological functions-such as visuomotor, cognitive, sensory, higher order, working memory and attention — this study aims to investigate whether these cytoarchitecturally different sub-regions have different functional connectivity to different functional brain networks.
\end{abstract}

Methods: This study examined 198 healthy subjects using resting-state fMRI and investigated the functional connectivity of seven sub-regions of the SPL to eight regional functional networks.

Results: The findings showed that most of the seven sub-regions were functionally connected to these targeted networks and that there are differences between these sub-regions and their functional connectivity patterns. The most consistent functional connectivity was observed with the visual and attention networks. There were also clear functional differences between Brodmann area (BA) 5 and BA7. BA5, with its three sub-regions, had strong functional connectivity to both the sensorimotor and salience networks.

Conclusion: These findings have enhanced our understanding of the functional organisations of the complexity of the SPL and its varied topology and also provide clear evidence of the functional patterns and involvements of the SPL in major brain functions.

Keywords: FMRI, Functional connectivity, Superior parietal cortex

\section{Key points}

- The SPL has a complex, heterogeneous topology that comprises more than seven sub-regions

- The functional connectivity of these sub-regions to different functional brain networks was investigated.

- There are similarities and differences between these sub-regions and their functional connectivity patterns.

*Correspondence: aaalahmadi@kau.edu.sa; adnan.alahmadi.11@ucl.ac.uk Department of Diagnostic Radiology, College of Applied Medical Science, King Abdulaziz University, Jeddah, Saudi Arabia
- The most consistent functional connectivity was observed with the visual and attention networks.

- There were also clear functional differences between Brodmann area (BA) 5 and BA7.

\section{Introduction}

The superior parietal lobule (SPL) plays an important role in different brain functions including visuomotor, cognitive, sensory, higher order, working memory and attentional [1-10]. Most of these findings were investigated using functional magnetic resonance imaging (fMRI) 
based on specific experimental tasks, and the findings reported relate to the SPL as a single region.

Another powerful and useful technique that aids the understanding of the brain's functional networking mechanism is resting-state fMRI (rsfMRI), which helps to determine the functional integrations of regions of the brain during rest [11]. rsfMRI is also a powerful tool regarding the structural understanding of cortical organisations, as emerging evidence from studies have shown that these functional, spontaneous low-frequency signals were significantly structurally correlated [12-14]. Evidence also suggests that these spontaneous fluctuations observed during rsfMRI produced functional networks that were continuously correlated with experimental tasks [13].

In addition, and in an effort to further understand the cortical organisation of the SPL, recent studies used cytoarchitecture analysis and probabilistic maps in ten post-mortem brains to analyse the SPL [15-17]. The studies showed that the SPL was sub-divided into eight sub-regions: $5 \mathrm{Ci}, 5 \mathrm{M}, 5 \mathrm{~L}, 7 \mathrm{PC}, 7 \mathrm{~A}, 7 \mathrm{P}, 7 \mathrm{M}$ and hIP3. Three of these sub-regions are located in Brodmann (BA) 5 , and four of them are located in BA7. Each of these subregions is different based on the receptor distribution patterns and the regional cytoarchitectonic properties.

The functional roles of each of these sub-regions have still not been fully investigated, and most of the previous studies mentioned above claimed the involvement of SPL as a single cortical region. The assumption is that since these sub-regions are different from an anatomical point of view, and each has different cytoarchitectonic properties, their differences can expect to be observed during functional connectivity to major network functions. Therefore, this study aims to use a large cohort of subjects to investigate the functional connectivity of each of these seven sub-regions of the SPL with major functional identified networks and to use rsfMRI to determine how these sub-regions differ based on functional connectivity.

\section{Methods}

\section{Subject recruitments and scanning}

A total of 198 healthy volunteer subjects were recruited for this study. Their ages ranged from 18 to 30, and 123 of the subjects were female. 171 of the subjects were right handed. The data were collected and downloaded from the Cambridge-Buckner dataset, which is part of the 1,000 Functional Connectomes Project (an open-access platform without any restrictions, https://www.nitrc.org/ projects/fcon_1000/) [12]. This is a non-restricted public dataset and is available to all. The IRP statement provided on the 1,000 Functional Connectomes Project website is shown on the following paragraph.
"The 1,000 Connectomes Project data-sharing effort will provide the research community with open access to datasets contributed by labs around the world. Datasets provided to the 1,000 Connectomes Project are to be de-identified prior to deposition of the data with the project (i.e. removal of any personal identifying information from header/support files). Upon arrival, datasets are automatically organised, and header files are replaced with novel header files to guarantee that any identifying personal information within the header or supporting files is removed. Prior to open-access sharing via webbased repository, all datasets will be further de-identified and anonymised by the removal of face information from the image to prevent any inappropriate reconstruction of the image that could lead to the identification of a participant. Furthermore, each participant's dataset will be assigned a randomised five-digit participant identifier, along with a site identifier (two letters which map to the site providing the data). The relationship between the anonymised code and the original subject ID will be destroyed to ensure that the dataset will be truly anonymised. For each dataset, demographic information provided via web-archive will be limited to (when available): age (18 and up), gender (male, female) and handedness. This information will serve to facilitate more careful characterisation of the data, without entailing a risk of violation of confidentiality. Datasets will only be included in the repository upon receipt of written expressed permission for usage of the dataset freely by the general public, without limitation."

This dataset was acquired using a typical rsfMRI protocol that is used by previous studies, see for review and references [18-20]. The registered clinical name for this project is the Cambridge-Buckner dataset. All of the 198 subjects were scanned using a Siemens 3-T Trim Trio scanner with the following parameters: $\mathrm{T} 2 *$ weighted Echo Planner Imaging (EPI) sequence with repetition time $(\mathrm{TR})=3 \mathrm{~s} ; \mathrm{TE}=30 \mathrm{~ms}$; number of slices $=47$ interleaved axial slices; voxel size: $3.0 \times 3.0 \times 3.0 \mathrm{~mm}^{3}$; number of time points (i.e. volumes) $=124$ volumes (the first 5 volumes were discarded). T1-weighted MPRAGE images with the following parameters: number of slices: 192; matrix size $144 \times 192$; voxel size: $1.20 \times 1.00 \times 1.33 \mathrm{~mm}^{3}$.

\section{Pre-processing}

The pre-processing and statistical analyses were performed using CONN and Statistical Parametric Mapping software (SPM12). The pre-processing of the rsfMRI imaging volumes included such typical pre-processing steps as slice timing corrections, realignment of the functional volumes, normalisation of the functional volumes to an MNI template using structural data, detection data outliers using the implanted artefact detection tools 
(ART) in CONN [21], and smoothing the functional volumes using an 8-mm kernel. In addition, in order to neutralise the effects of artefacts and confounds (e.g. white matter, CSF signals, and motion and scrubbing parameters) from the BOLD signal, temporal processing with data denoising was also applied.

\section{Selection of the regions of interest}

The seeds identified in this study were the seven subregions of the SPL, namely the $\mathrm{BA} 5$ sub-regions $(5 \mathrm{Ci}$, $5 \mathrm{M}$, and $5 \mathrm{~L}$ ) and the BA7 sub-regions (7PC, 7A, 7P, and $7 \mathrm{M}$ ) (Fig. 1). These regions were identified using the cytoarchitectonic probability anatomy map, which is guided by the cytoarchitectonic properties of these regions based on the data from ten post-mortem brains $[15,17,22,23]$. The target brain regions were major collections of functional networks, as defined in CONN and shown in Table 1. In addition to the default mode, the functional networks studied were attention, sensorimotor, visual, salience, dorsal attention, frontoparietal, cerebellar, and language. This atlas of commonly used networks was defined based on an independent component analysis (ICA) of the human connectivity project using 497 subjects.

\section{Statistical analysis}

Statistical analysis was calculated at two levels. At the first level (subject-level), weighted general linear bivariate correlation models, including regions of interests (ROI)-to-ROI connectivity matrices between the

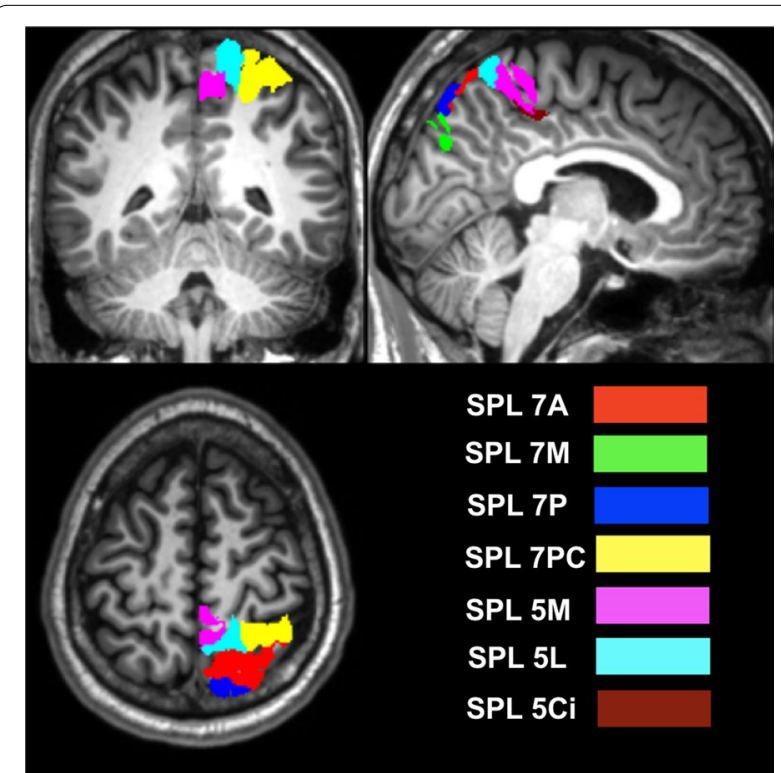

Fig. 1 Illustrations of the seven SPL source regions used in this study in the right hemisphere
Table 1 Targeted networks with the regions that comprise them. Also shown the coordinates of these regions

\begin{tabular}{|c|c|}
\hline Network & Regions \\
\hline \multirow[t]{4}{*}{ Default mode } & $\operatorname{MPFC}(1,55,-3)$ \\
\hline & $\operatorname{LP}(\mathrm{L})(-39,-77,33)$ \\
\hline & LP (R) $(47,-67,29)$ \\
\hline & $\operatorname{PCC}(1,-61,38)$ \\
\hline \multirow[t]{3}{*}{ Sensorimotor } & Lateral $(\mathrm{L})(-55,-12,29)$ \\
\hline & Lateral (R) $(56,-10,29)$ \\
\hline & Superior $(0,-31,67)$ \\
\hline \multirow[t]{4}{*}{ Visual } & Medial $(2,-79,12)$ \\
\hline & Occipital $(0,-93,-4)$ \\
\hline & Lateral $(\mathrm{L})(-37,-79,10)$ \\
\hline & Lateral (R) $(38,-72,13)$ \\
\hline \multirow[t]{7}{*}{ Salience } & $\operatorname{ACC}(0,22,35)$ \\
\hline & Insula (L) $(-44,13,1)$ \\
\hline & Insula (R) $(47,14,0)$ \\
\hline & $\operatorname{RPFC}(\mathrm{L})(-32,45,27)$ \\
\hline & $\operatorname{RPFC}(\mathrm{R})(32,46,27)$ \\
\hline & SMG (L) $(-60,-39,31)$ \\
\hline & $\operatorname{SMG}(\mathrm{R})(62,-35,32)$ \\
\hline \multirow[t]{4}{*}{ Dorsal Attention } & $\operatorname{FEF}(L)(-27,-9,64)$ \\
\hline & FEF (R) $(30,-6,64)$ \\
\hline & IPS (L) $(-39,-43,52)$ \\
\hline & IPS (R) $(39,-42,54)$ \\
\hline \multirow[t]{4}{*}{ Fronto-Parietal } & $\operatorname{LPFC}(\mathrm{L})(-43,33,28)$ \\
\hline & PPC (L) $(-46,-58,49)$ \\
\hline & LPFC (R) $(41,38,30)$ \\
\hline & PPC (R) $(52,-52,45)$ \\
\hline \multirow[t]{4}{*}{ Language } & IFG (L) $(-51,26,2)$ \\
\hline & IFG (R) $(54,28,1)$ \\
\hline & pSTG (L) $(-57,-47,15)$ \\
\hline & pSTG (R) $(59,-42,13)$ \\
\hline \multirow[t]{2}{*}{ Cerebellar } & Anterior $(0,-63,-30)$ \\
\hline & Posterior $(0,-79,-32)$ \\
\hline
\end{tabular}

Abbreviation: MPFC: medial prefrontal cortex; LP: lateral parietal; PCC: posterior cingulate cortex; ACC: anterior cingulate cortex; RPFC: rostrolateral prefrontal cortex; SMG: supramarginal gyrus; FEF: frontal eye fields; IPS: intraparietal sulcus; LPFC: lateral prefrontal cortex; PPC: posterior parietal cortex; LPFC: lateral prefrontal cortex; IFG: inferior frontal gyrus; PSTG: posterior superior temporal gyrus

pre-defined ROIs, were calculated for each subject. These connectivity matrices were defined as Fishertransformed bivariate correlation coefficient between the pair of ROI timeseries. At the second level, functional connectivity measures were calculated and compared using group-level statistical analysis, such as T-tests and/or F-tests where appropriate, and identifying and comparing the rsfMRI networks connected to each of the sub-regions of the SPC at the group level. The standard setting for the results is displayed using 
a corrected false discovery rate (FDR) $(\mathrm{p}<0.05)$ (multivariate statistics parametric (MVPA) omnibus test) [21]. Here, cluster-level inferences are determined using functional network connectivity multivariate parametric statistic inferences by considering groups or networks of related ROIs. Then, the analysis is done by analysing the entire set of connections between the ROIs in terms of within- and between-network connectivity [24]. This method effectively performs a multivariate parametric general linear model analysis for all connections. The resultant map is of F-statistical tests for each pair of networks. The FDR cluster level is defined as the expected proportion of false discoveries among all pairs of networks with similar or larger effects across the entire set of functional connectivity network pairs [25]. One of the reasons for using the FDR over family wise error (FWE) is that the FDR is more sensitive in controlling peaks, with minimal cost of false positives [26]. For additional details, please refer to the above papers or the CONN website (https:// web.conn-toolbox.org).

\section{Results}

All of the sub-regions are connected to each other, and the functional connectivity between the seven subregions of the SPL is shown in Fig. 2. Figure 2 also shows that the strength of each of the connections is statistically different from each other.

The study investigated the functional connectivity between the seven sub-regions of the SPC in the two hemispheres of the brain to the eight targeted networks. In general terms, the results showed that there are strong connections (either positive or negative) between the sub-regions and the targeted networks. There were similarities and differences among the connections, which are summarised in Figs. 3 and 4. Additionally, Fig. 5 summarises the connections by indicating whether there is a positive connection, a negative connection, or no connection. The key findings are shown below.

\section{Functional connectivity with the default mode network}

In general, the seven sub-regions of the SPL had positive connections with the regions defining the default mode network, particularly the lateral parietal (LP) and

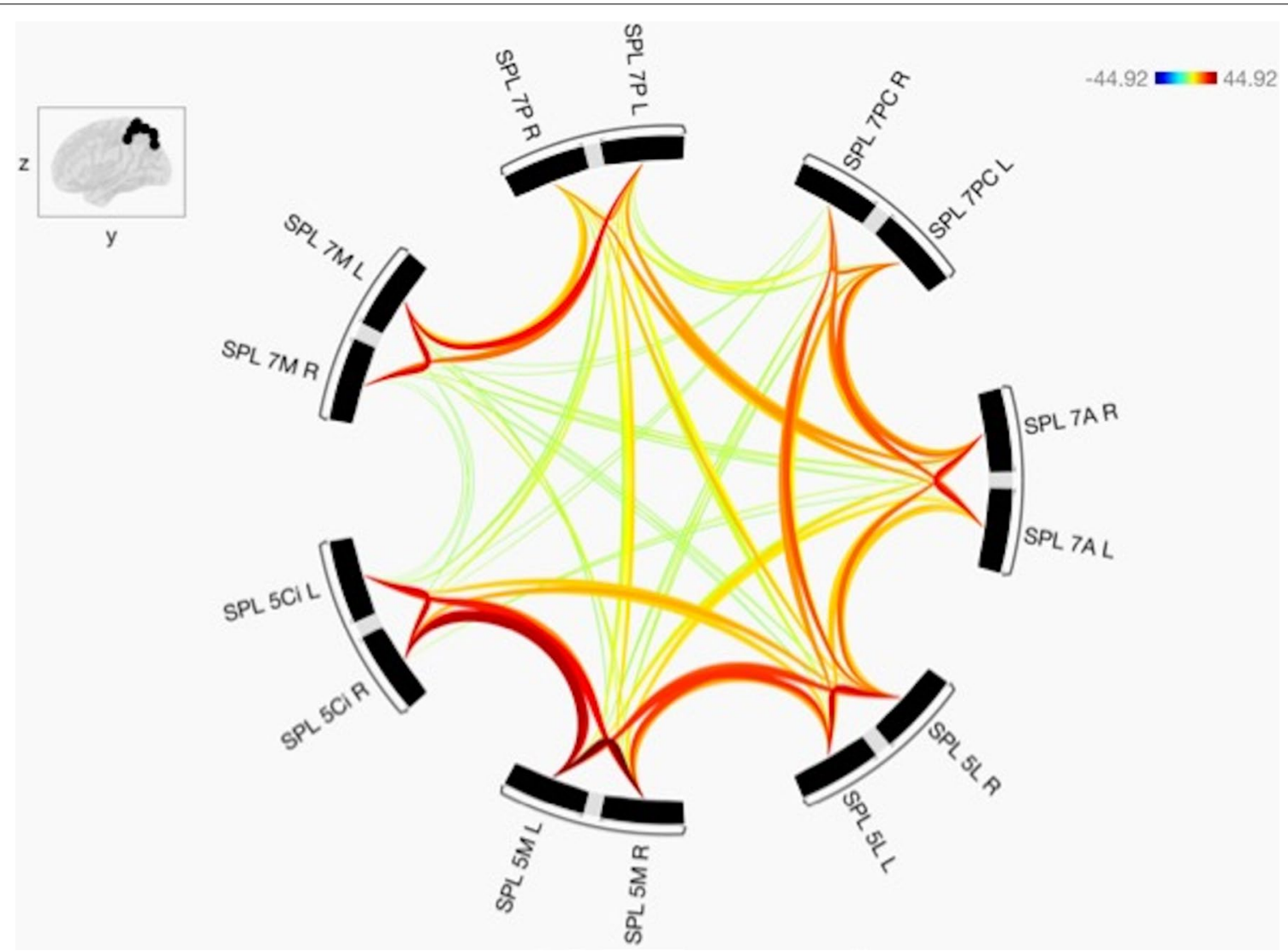

Fig. 2 The functional connectivity among the sub-regions of the SPL is shown. These are the seven investigated sub-regions of the SPL in right and left hemispheres (14 sub-regions). The lines of the connections in red indicate positive connectivity, and these colours are proportional to statistical strength. Additionally, the T-bar is shown in the top right-hand corner 

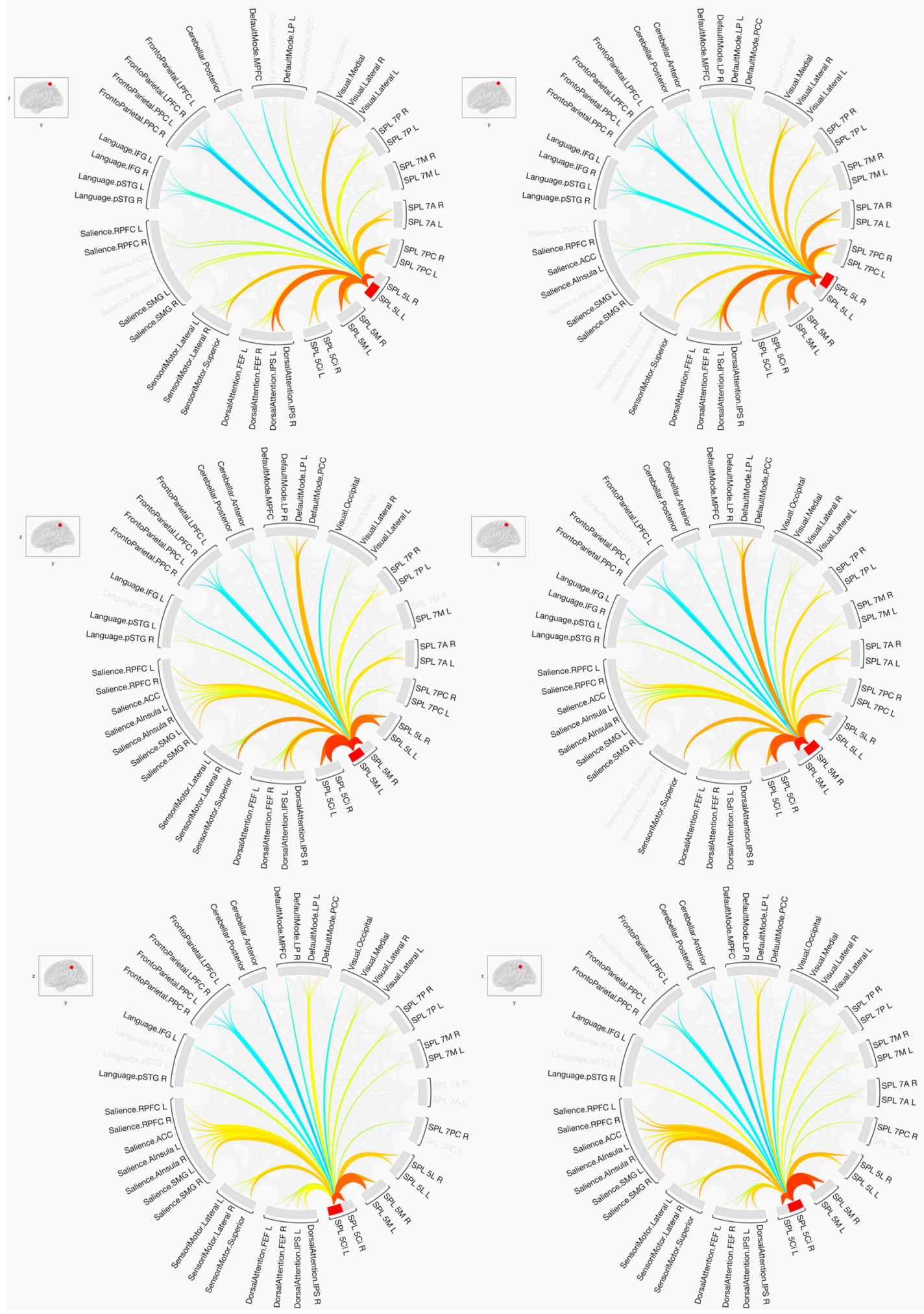

Fig. 3 The functional connectivity of the three SPL sub-regions of BA5 in both hemispheres is shown here. The lines of the connections in red indicate positive connectivity while blue indicates negative connectivity. The colours of the lines are proportional to statistical strength, and the T-bar is shown in the top right-hand corner 

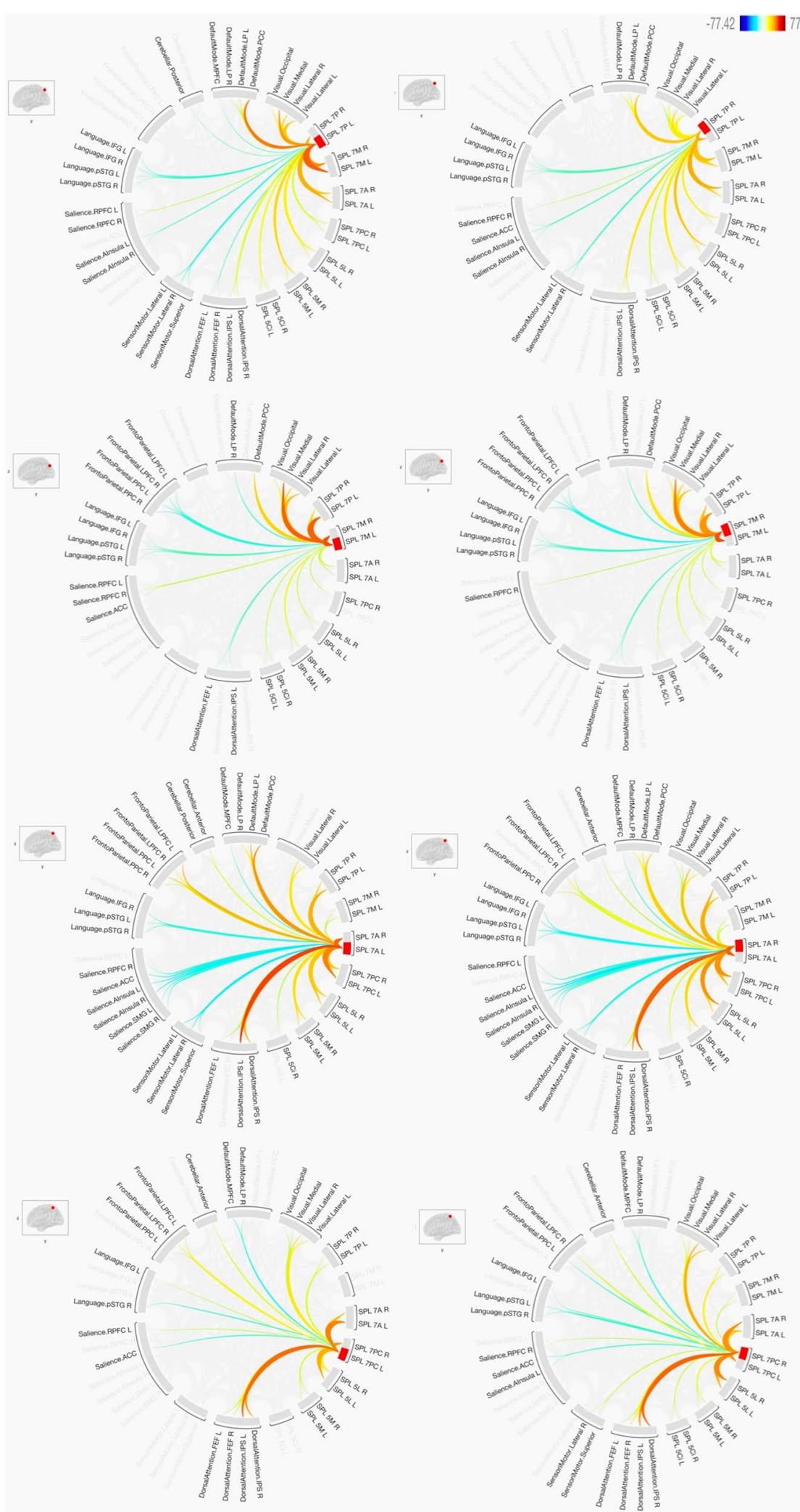

Fig. 4 The functional connectivity of the four SPL sub-regions of BA 7 in both hemispheres is shown here. The lines of the connections in red indicate positive connectivity while blue indicates negative connectivity. The colours of the lines are proportional to statistical strength, and the T-bar is shown in the top right-hand corner 


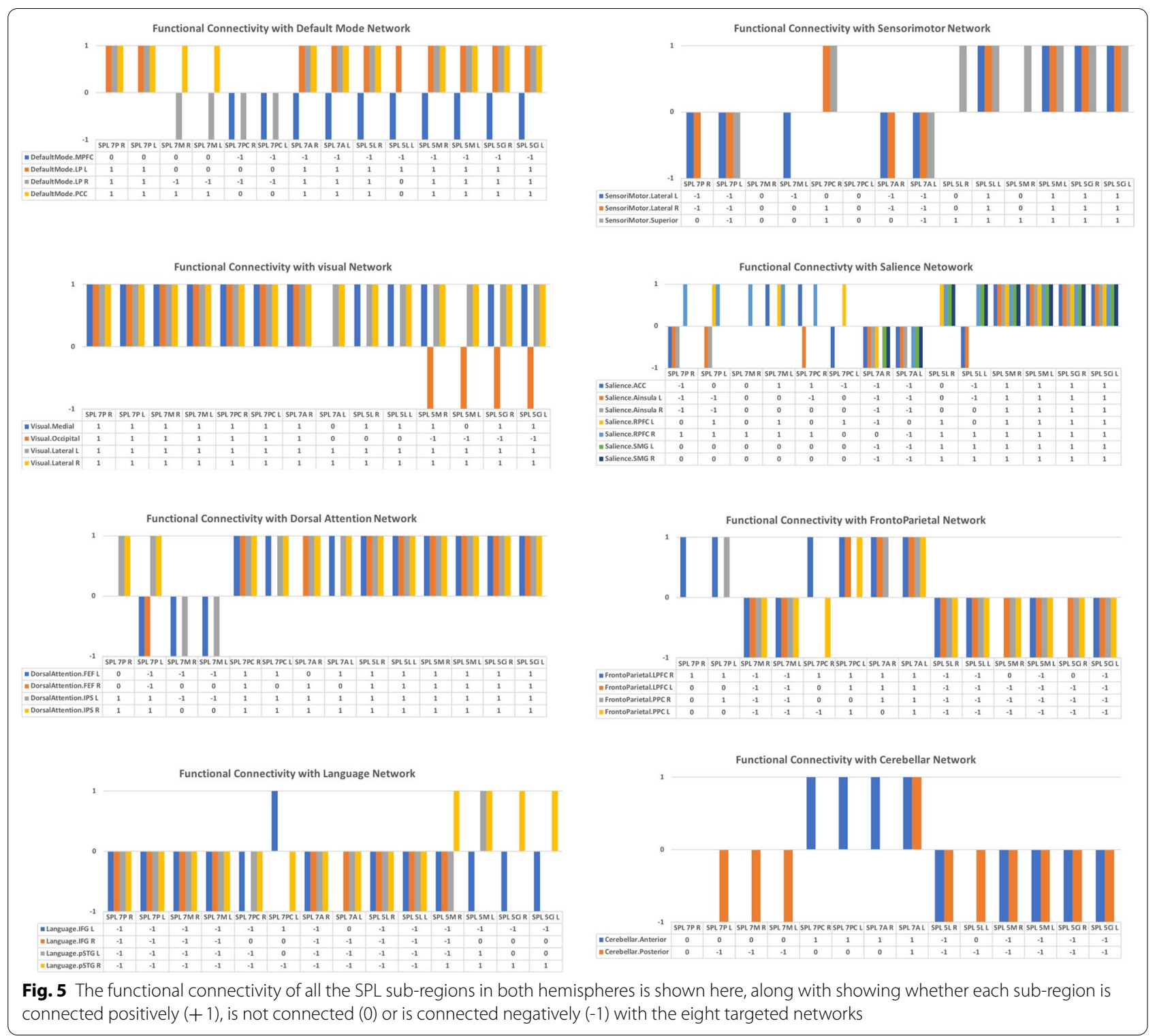

posterior cingulate cortex (PCC). Negative connections were also observed, particularly with the medial prefrontal cortex (MPFC) and within BA5. The results also indicated that there were no significant differences between the two hemispheres as most of the connections to these sub-regions of the SPL were identical. The sub-regions that had clear and distinct differences from the other sub-regions were the SPL $7 \mathrm{M}$, the SPL 7PC and the left SPL 5L.

\section{Functional connectivity with the sensorimotor network}

In general, most of the sub-regions of the SPL located within BA7 had negative connections with the subregions defining the sensorimotor network, while all the sub-regions of the SPL located within BA5 had positive connections. In contrast, the right SPL $7 \mathrm{M}$ and the left SPL 7PC had no connections at all.

\section{Functional connectivity with the visual network}

In general, most of the sub-regions of the SPL had positive connections with the regions defining the visual network. This was highly constant within the sub-regions of BA7. Within the sub-regions of BA5, there were negative connections with the SPL $5 \mathrm{M}$ and SPL $5 \mathrm{Ci}$.

\section{Functional connectivity with the salience network}

There were clear differences between the sub-regions of the SPL within BA7 and the regions defining the salience 
network. These were either defined by positive, negative or no connections at all. While the sub-regions of SPL within BA5 had mostly positive connections, the sub-regions of the SPL within BA7 contained differences between the two hemispheres when functional connectivity with the salience network was investigated. The largest number of positive connections of the SPL BA7 sub-regions were seen with the rostrolateral prefrontal cortex (RPFC) of the salience network, while the SPL 7A had negative connections with most of the regions making up the salience network.

\section{Functional connectivity with the dorsal attention network} In general, most of the sub-regions of the SPL had positive connections with the regions defining the dorsal attention network. This was highly constant within the sub-regions of BA5. Within the sub-regions of BA7, there were negative connections with the SPL $7 \mathrm{M}$ and left SPL 5P. SPL 7P only had positive connections with the intraparietal sulcus (IPS) of the dorsal attention network, while the left SPL 7P had negative connections with the frontal eye fields (FEF) of the dorsal attention network. The SPL $7 \mathrm{M}$ had negative connections in both hemispheres with the FEF and IPS of the dorsal attention network, while it had no connections with the IPS.

\section{Functional connectivity with the frontoparietal network}

In general, most of the sub-regions of the SPL had negative connections with the regions making up the frontoparietal network. These negative connections were constant within the sub-regions of BA5. The sub-regions of BA7 were heterogeneous in terms of their functional connections with the regions defining the frontoparietal network, while SPL $7 \mathrm{M}$ had negative connections. Positive connections were only observed in SPL 7P with the right lateral prefrontal cortex (LPFC) and the posterior parietal cortex (PPC) of the frontoparietal network. Hemispheric differences were observed in SPL 7PC, with the right SPL 7PC having a negative connection with the left PPC of the frontoparietal network.

\section{Functional connectivity with the language network}

In general, most of the sub-regions of the SPL had negative connections with the regions defining the language network. These negative connections were mostly within the sub-regions of BA7. The only region that had a positive connection with the language network within BA7 was the left SPL 7PC, which had a positive connection with the left inferior frontal gyrus (IFG). The sub-regions of BA5 were partially heterogeneous in terms of their functional connections with the regions defining the language network. Two sub-regions of BA5-SPL $5 \mathrm{M}$ and SPL $5 \mathrm{Ci}-$ had positive connections with the right posterior superior temporal gyrus (pSTG) of the language network. In contrast, the only negative connections in these two sub-regions were with the left IFG. Hemispheric differences were not significantly observed among all of the sub-regions and it was mainly related to the SPL $5 \mathrm{M}$ and SPL $5 \mathrm{Ci}$.

\section{Functional connectivity with the cerebellar network}

There is a clear difference between the sub-regions of SPL within BA7 and the regions defining the cerebellar network, with heterogeneous functional connectivity within the BA7 sub-regions being observed. Negative connections with the posterior cerebellum were constant with all the sub-regions of BA5, while negative connections were also observed with the SPL $7 \mathrm{M}$ and the left SPL 7P. The anterior cerebellum also showed negative connections with most of the sub-regions of BA5. Positive connections were mainly observed between the anterior cerebellum and the SPL 7PC and SPL 7A.

\section{Discussion}

This study used a large dataset to investigate the functional connectivity of seven sub-regions of the SPL with eight regional network functions using rsfMRI. These seven sub-regions were shown to be different from each other both structurally and cytoarchitecturally [15-17, $23,27]$. The assumption was that since these sub-regions were cytoarchitecturally different, their functional connectivity could also be different. In general terms, the findings of this study showed that these sub-regions were connected to the eight functional networks. The functional connectivity was similar between some networks and different between other networks.

The findings showed the important functional role that the SPL plays in terms of its involvements in different functional domains, particularly regarding the attentional and visual pathways. Here, the functional connectivity was most strongly connected with the networks in most of the sub-regions of the SPL. This is in line with previous findings that showed a strong involvement of the SPL with visual and attentional functions [2, 8, 28-30]. However, the connections to the visual and attention networks were neither homogeneous nor consistent among the sub-regions of the SPL.

The results of this study indicated that all of the subregions of BA7 were significantly involved with the visual networks, whereas the sub-regions of BA5 were showing some heterogeneous functional connections. For example, there were no relationships between the sub-regions of BA5 with the visual occipital part of the visual network. This visual occipital part was shown to be mainly involved in the perception of visual shapes [13]. 
This could indicate that not all of the SPL is involved in visual functions. SPL BA7 plays a greater role in visual processing, while SPL BA5 has limited involvements in visual functions but no direct involvement in the perception of visual shapes. When looking at the attentional network, which consisted of FEF and IPS, all of the subregions of SPL BA5 were directly involved in attentional functions to a greater extent than the sub-regions of SPL BA7. There was heterogeneous connectivity within the sub-regions of SPL BA7 where only the right SPL 7PC had full connectivity with all of the sub-regions of the attention network. Some studies have shown that the involvement of SPL in attentions was predominant in the right hemisphere [9], which could indicate that this is only true within SPL BA7 but not within SPL BA5.

In addition, the sensorimotor network involves areas that are related to motor and sensory functions, including the pre- and post-central gyri as well as the supplementary motor areas. Indeed, the SPL plays an important role in sensorimotor tasks, particularly visuomotor tasks or tasks that require certain visuomotor attention [8].

The results of this study showed that there were distinct differences between BA5 and BA7. Most of the sub-regions of BA5 had positive connectivity with the sensorimotor networks, particularly the superior region, whereas the BA7 sub-regions, except for SPL 7PC, had either negative or no connections with the sensorimotor network. Previous studies have shown that BA5 has extensive involvement in sensorimotor functions, while BA7 has less involvement $[31,32]$. The results indicated that most of the sub-regions of BA5, apart from the right SPL $5 \mathrm{M}$, were indeed involved with the sensorimotor network.

The results also showed that the right SPL 7PC is the only sub-region in BA7 that was involved with the sensorimotor network, indicating that there are heterogeneous connections in the sub-regions of BA5 and BA7. However, these heterogeneous connections were only found within one of the sub-regions.

The default mode network is a network of interacting brain regions that include the MPFC, the LP and the PCC. These interacting brain regions are related to each other and usually have distinct patterns compared to other networks [33]. Typically, the default mode network is related to intrinsic changes and it has greater remarkable differences in neurological diseases [34-36]. In our study, the seven sub-regions of the SPL had mostly positive connections with the regions defining the default mode network, particularly with the LP and PCC of the default mode network. These findings indicate the importance of these sub-regions in their connections to the default mode network and indicate how the default mode network may play a role in the brain functions controlled by the SPL. The fact that these sub-regions had more positive connections with the LP and PCC of the default mode network could be because of their involvement in higher-order cognitive and attentional tasks.

However, the results also showed that the default mode network had negative connections with the MPFC. This suggests that the default mode and the seven sub-regions of the SPL have some heterogeneous relationships and that the default mode network sub-regions should be investigated as a heterogeneous network. This is in line with studies that showed that the prefrontal cortical part, which had negative connections with most of the SPL sub-regions, and the PCC, which had positive connections with most of the SPL sub-regions, are distinctly different from each other [37].

Our study also showed that there were sub-regions of the SPL that were distinctly different from the other subregions, namely the SPL $7 \mathrm{M}$, SPL 7PC and the left SPL 5L. Recent studies showed that the SPL $7 \mathrm{M}$, SPL 7PC and SPL 5L showed distinct functional activations or connectivity during different operational tasks [1, 38], which could explain their distinct patterns of connections with the prefrontal part of the default mode network.

Moreover, the involvement of the SPL's sub-regions with the other remaining networks-namely the salience, frontoparietal, language and cerebellar networks-were heterogeneous and were not strongly connected with all of the sub-regions. In addition, anticorrelation or negative connectivity was often observed between most of the sub-regions of the SPL with these networks. However, the interpretation of these negative functional connections is beyond the scope of this study, particularly as there is still debate regarding the meaning of these anticorrelation signals in functional connectivity [39]. The results of this study could indicate that heterogeneous functional connectivity is present and that the SPL should not be seen or investigated as a whole region of interest.

Two further observations are worth mentioning and highlighting. SPL 7PC and SPL 7A were the two subregions found to be positively connected to the anterior cerebellum, which is known to be involved in motorrelated functions [40-46]. This suggests that these two sub-regions could have a direct role in organising the pathways and functional involvements of the anterior cerebellum. Also, recent studies have found that SPL 7PC was mainly involved in execution and motor functions [1], which could explain the direct involvement of this sub-region as a motor pathway with the anterior cerebellum. Finally, this study also showed that the SPL BA5 sub-regions were all involved and correlated positivity with the salience network. The salience network is known to be involved in salience functions and attentions [47], 
which could also suggest that BA5 has a role in driving the pathways of focused and salience functions.

\section{Methodological and future considerations}

Despite the coherent findings in this study and the clear differences among the SPL sub-regions, there are some potential limitations that should be considered in future studies. Future studies could investigate the question of the differences of the functional connectivity of these sub-regions using high-resolution data (i.e. $1 \mathrm{~mm} 3$ ). This may help to enhance the subtle differences among these sub-regions. Usually, such high-resolution data are acquired using high field scanner strength (i.e. $7 \mathrm{~T}$ ).

In addition, a potential limitation of this study was the applied smoothing kernel. Although $8 \mathrm{~mm} 3$ is a default (and considered typical [48-51]) kernel for such voxel resolution, future studies may consider the effects of smoothing on the connectivity of these sub-regions. However, some recent studies have shown that smoothing kernels could have little effects, especially on rs-fMRI ROI to ROI analysis [52, 53]. Regardless of these studies and since this study's source regions of interest were adjacent, one could argue that the $8 \mathrm{~mm} 3$ smoothing kernel would blur those regions and affect the results. Therefore, a minimal smoothing kernel (or no smoothing) could be tested in future studies to compare and validate the results.

Another limitation of this study is the usage of an open-access dataset. The limitation is related to the information that could be used in the statistical method to investigate, for example, if gender and education level could be confounding factors that may have affected the results. Previous studies have shown functional connectivity based on gender differences [54]; however, such factors need careful data acquisition and planning before starting studies, and performing such was beyond the aim of this study. In addition, handedness in this study was shown to have no effect on the investigated results, when used as a covariate in the GLM. This finding is likely because the number of right-handed subjects was large compared to left-handed subjects, and this study did not aim to investigate the effect of handedness on the results. Thus, future studies could design a study to investigate such effects, which probably could affect the functional connectivity of SPL 7A and $5 \mathrm{M}$ with other brain regions.

In addition, one of this study's limitations is the analysed dataset's parameters. For example, this study's number of time points is not large. Longer scans (and more time points) can increase resting state reliability and improve sensitivity $[55,56]$. Therefore, future studies could consider better quality datasets to check the reliability of this study's findings.
Finally, future studies could investigate structural and functional connectivity among those sub-regions to clarify the unexplained connectivity and better understand the physiological organisations underlying the regions with different networks. For example, it may be that the internal interactions spontaneous patterns could be highly structured $[1,27,57,58]$. Or these functional connections could not be correlated structurally, which may suggest mediations by indirect structural connections [58]. Also, future studies could investigate effective connectivity and task-related activations of these sub-regions [59]. Therefore, a multimodal approach to analyse these regions is needed. For example, a recent study aimed to identify a convergent organisation of the SPL using structural and functional connectivity [1]. The researchers identified five sub-regions (two anterior and three posterior) in the SPL based on multimodal neuroimaging analyses. They were able to show functional-related differences of the identified regions of the brain that were linked to vision, motor control, perception, memory and attention. The findings of their results are in line with the findings of our study in terms of the different functional involvements of the SPL regions. One could therefore combine the usage of these cytoarchitecture identified sub-regions and with structurally identified approaches to further enhance our understanding of the physiological organisations of these sub-regions.

Therefore, it must also be acknowledged that interpreting the specific connectivity patterns of these sub-regions is difficult to do. This is because, on the one hand, different task experimental fMRI needs to be implemented to test for the involvement of these sub-regions in different brain functions. On the other hand, this study is one of the first that attempts to understand the cortical heterogeneous functional connectivity of these cytoarchitecturally different sub-regions.

However, the findings of this study are important because they provide clear evidence that in addition to the cytoarchitectural differences among these seven SPL sub-regions, there are also functional connectivity differences that should also be considered. Investigating the similarities and differences in functional connectivity among these seven sub-regions of the SPL with important targeted functional brain networks should highlight the importance of using more specified detailed anatomical atlases to investigate these cortical regions in more specific ways. It could also help future studies to focus on the impacts of different neurodegenerative diseases, and how the pathologies of such diseases affect the functional connectivity of these sub-regions. The findings of this study could also enhance our understanding of the functional organisations of the complexity of the SPL and its varied 
topology and could also assist in reviewing the applied specific task functional activation involvements of these sub-regions.

\section{Conclusion}

This study has shown the similarities and differences in functional connectivity between the seven cytoarchitecturally different sub-regions of the SPL with different functional brain networks. The study has shown how each of the SPL sub-regions plays an important role in visual and attentional functions. The study has also shown the various involvements of the subregions of BA7 and BA5 in other brain functions. The heterogeneity of the functional connectivity of these sub-regions has also been proven by this study, which suggests that the SPL has different complex structural and functional topological organisations that should always be considered when investigating the physiology of this important cortical region, or in neurological disease applications.

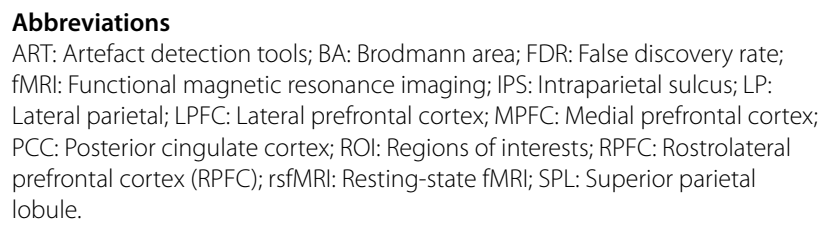

Abbreviations

ART: Artefact detection tools; BA: Brodmann area; FDR: False discovery rate; fMRI: Functional magnetic resonance imaging; IPS: Intraparietal sulcus; LP: Lateral parietal; LPFC: Lateral prefrontal cortex; MPFC: Medial prefrontal cortex; PCC: Posterior cingulate cortex; ROI: Regions of interests; RPFC: Rostrolateral prefrontal cortex (RPFC); rsfMRl: Resting-state fMRl; SPL: Superior parietal lobule.

\section{Authors' contributions}

All authors read and approved the final manuscript.

\section{Funding}

This research work was funded by institutional fund projects under grant nos. (IFPHI-327-142-2020) \& (IFPHI-095-142-2020) \& (IFPRC-004-142-2020) \& (J: 67-142-1441). Therefore, authors gratefully acknowledge technical and financial support from the ministry of education and King Abdulaziz University, Deanship of Scientific Research (DSR), Jeddah, Saudi Arabia.

\section{Availability of data and materials}

All data used in the study are available in this website (https://www.nitrc.org/ projects/fcon_1000/).

\section{Declarations}

\section{Ethics approval and consent to participate}

The data sample were taken from the Cambridge-Buckner data sample (open access) (https://www.nitrc.org/projects/fcon_1000/). All procedures performed in the studies involving human participants were in accordance with the ethical standards of the institutional and/or national research committee and with the 1964 Helsinki Declaration and its later amendments or comparable ethical standards.

\section{Consent for publication}

Informed consent was obtained from all individual participants included in the study. The included participants data were taken from the CambridgeBuckner data sample, which is part of the 1000 functional connectomes project.

\section{Competing interests}

The authors declare that they have no conflict of interest.
Received: 13 January 2021 Accepted: 23 March 2021

Published online: 13 April 2021

\section{References}

1. Wang J et al (2015) Convergent functional architecture of the superior parietal lobule unraveled with multimodal neuroimaging approaches. Hum Brain Mapp 36(1):238-257

2. Culham JC, Valyear KF (2006) Human parietal cortex in action. Curr Opin Neurobiol 16(2):205-212

3. Weiss PH et al (2003) Are action and perception in near and far space additive or interactive factors? Neuroimage 18(4):837-846

4. Vingerhoets $\mathrm{G}$ et al (2002) Motor imagery in mental rotation: an fMRI study. Neuroimage 17(3):1623-1633

5. Zago L, Tzourio-Mazoyer N (2002) Distinguishing visuospatial working memory and complex mental calculation areas within the parietal lobes. Neurosci Lett 331(1):45-49

6. Wenderoth $\mathrm{N}$ et al (2004) Parieto-premotor areas mediate directional interference during bimanual movements. Cereb Cortex 14(10):1153-1163

7. Bray S et al (2013) Structural connectivity of visuotopic intraparietal sulcus. Neuroimage 82:137-145

8. Corbetta M et al (1995) Superior parietal cortex activation during spatial attention shifts and visual feature conjunction. Science 270(5237):802-805

9. Coull J, Frith C (1998) Differential activation of right superior parietal cortex and intraparietal sulcus by spatial and nonspatial attention. Neuroimage 8(2):176-187

10. Lloyd D, Morrison I, Roberts N (2006) Role for human posterior parietal cortex in visual processing of aversive objects in peripersonal space. J Neurophysiol 95(1):205-214

11. Biswal B et al (1995) Functional connectivity in the motor cortex of resting human brain using echo-planar MRI. Magn Reson Med 34(4):537-541

12. Biswal BB et al (2010) Toward discovery science of human brain function. Proc Natl Acad Sci 107(10):4734-4739

13. Smith SM et al (2009) Correspondence of the brain's functional architecture during activation and rest. Proc Natl Acad Sci 106(31):13040-13045

14. Smith SM et al (2013) Functional connectomics from resting-state fMRI. Trends Cogn Sci 17(12):666-682

15. Scheperjans F et al (2008) Probabilistic maps, morphometry, and variability of cytoarchitectonic areas in the human superior parietal cortex. Cereb Cortex 18(9):2141-2157

16. Scheperjans F et al (2005) Subdivisions of human parietal area 5 revealed by quantitative receptor autoradiography: a parietal region between motor, somatosensory, and cingulate cortical areas. Neuroimage 25(3):975-992

17. Scheperjans F et al (2005) Transmitter receptors reveal segregation of cortical areas in the human superior parietal cortex: relations to visual and somatosensory regions. Neuroimage 28(2):362-379

18. Dijk, K.R.a.V., et al (2010) Intrinsic functional connectivity as a tool for human connectomics: theory. Properties Optim 02138:297-321

19. O'Rawe JF, Ide JS, Leung H-C (2019) Model testing for distinctive functional connectivity gradients with resting-state fMRI data. Neuroimage 185:102-110

20. Murphy K, Bodurka J, Bandettini PA (2007) How long to scan? The relationship between $\mathrm{fMRI}$ temporal signal to noise ratio and necessary scan duration. Neuroimage 34(2):565-574

21. Whitfield-Gabrieli S, Nieto-Castanon A (2012) Conn: a functional connectivity toolbox for correlated and anticorrelated brain networks. Brain connectivity 2(3):125-141

22. Eickhoff SB et al (2005) A new SPM toolbox for combining probabilistic cytoarchitectonic maps and functional imaging data. Neuroimage 25(4):1325-1335

23. Scheperjans F et al (2008) Observer-independent cytoarchitectonic mapping of the human superior parietal cortex. Cereb Cortex 18(4):846-867

24. Jafri MJ et al (2008) A method for functional network connectivity among spatially independent resting-state components in schizophrenia. Neuroimage 39(4):1666-1681 
25. Benjamini Y, Hochberg Y (1995) Controlling the false discovery rate: a practical and powerful approach to multiple testing. J Roy Stat Soc: Ser B (Methodol) 57(1):289-300

26. Chumbley J et al (2010) Topological FDR for neuroimaging. Neuroimage 49(4):3057-3064

27. Mars RB et al (2011) Diffusion-weighted imaging tractography-based parcellation of the human parietal cortex and comparison with human and macaque resting-state functional connectivity. J Neurosci 31(11):4087-4100

28. Behrmann M, Geng JJ, Shomstein S (2004) Parietal cortex and attention. Curr Opin Neurobiol 14(2):212-217

29. Corbetta M et al (1993) A PET study of visuospatial attention. J Neurosci 13(3):1202-1226

30. Shulman GL et al (2010) Right hemisphere dominance during spatial selective attention and target detection occurs outside the dorsal frontoparietal network. J Neurosci 30(10):3640-3651

31. Li G et al (2004) Cortical activations upon stimulation of the sensorimotor-implicated acupoints. Magn Reson Imaging 22(5):639-644

32. Premji A, Rai N, Nelson A (2011) Area 5 influences excitability within the primary motor cortex in humans. PLoS ONE 6:5

33. Raichle ME (2015) The brain's default mode network. Annu Rev Neurosci 38:433-447

34. Greicius MD et al (2004) Default-mode network activity distinguishes Alzheimer's disease from healthy aging: evidence from functional MRI Proc Natl Acad Sci 101(13):4637-4642

35. Uddin LQ et al (2008) Network homogeneity reveals decreased integrity of default-mode network in ADHD. J Neurosci Methods 169(1):249-254

36. Whitfield-Gabrieli S, Ford JM (2012) Default mode network activity and connectivity in psychopathology. Annu Rev Clin Psychol 8:49-76

37. Uddin LQ et al (2009) Functional connectivity of default mode network components: correlation, anticorrelation, and causality. Hum Brain Mapp 30(2):625-637

38. Rosenberg-Lee $M$ et al (2011) Functional dissociations between four basic arithmetic operations in the human posterior parietal cortex: a cytoarchitectonic mapping study. Neuropsychologia 49(9):2592-2608

39. Chen $\mathrm{G}$ et al (2011) Negative functional connectivity and its dependence on the shortest path length of positive network in the resting-state human brain. Brain Connectivity 1(3):195-206

40. Alahmadi AA et al (2016) Complex motor task associated with non-linear BOLD responses in cerebro-cortical areas and cerebellum. Brain Struct Funct 221(5):2443-2458

41. Alahmadi AA et al (2017) Cerebellar lobules and dentate nuclei mirror cortical force-related-BOLD responses: Beyond all (linear) expectations. Hum Brain Mapp 38(5):2566-2579

42. Alahmadi AA et al (2015) Differential involvement of cortical and cerebellar areas using dominant and nondominant hands: An FMRI study. Hum Brain Mapp 36(12):5079-5100
43. Cui SZ et al (2000) Both sides of human cerebellum involved in preparation and execution of sequential movements. NeuroReport 11(17):3849-3853

44. Imamizu H et al (2000) Human cerebellar activity reflecting an acquired internal model of a new tool. Nature 403(6766):192-195

45. Schlerf, J., et al., Big challenges from the little brain-imaging the cerebellum. Advanced brain neuroimaging topics in health and disease-methods and applications, 2014.

46. Stoodley CJ (2012) The cerebellum and cognition: evidence from functional imaging studies. Cerebellum (London, England) 11(2):352-365

47. Menon V, Uddin LQ (2010) Saliency, switching, attention and control: a network model of insula function. Brain Struct Funct 214(5-6):655-667

48. Strother SC (2006) Evaluating fMRI preprocessing pipelines. IEEE Eng Med Biol Mag 25(2):27-41

49. Wang J et al (2005) To smooth or not to smooth? ROC analysis of perfusion fMRI data. Magn Reson Imaging 23(1):75-81

50. Hagler DJ Jr, Saygin AP, Sereno MI (2006) Smoothing and cluster thresholding for cortical surface-based group analysis of fMRI data. Neuroimage 33(4):1093-1103

51. Wu CW et al (2011) Empirical evaluations of slice-timing, smoothing, and normalization effects in seed-based, resting-state functional magnetic resonance imaging analyses. Brain connectivity 1(5):401-410

52. Alahmadi AA (2020) Effects of different smoothing on global and regional resting functional connectivity. Neuroradiology 2020:1-11

53. Molloy EK, Meyerand ME, Birn RM (2014) The influence of spatial resolution and smoothing on the detectability of resting-state and task fMRI. Neuroimage 86:221-230

54. Filippi M et al (2013) The organization of intrinsic brain activity differs between genders: A resting-state fMRI study in a large cohort of young healthy subjects. Hum Brain Mapp 34(6):1330-1343

55. Birn RM et al (2013) The effect of scan length on the reliability of restingstate fMRI connectivity estimates. Neuroimage 83:550-558

56. Noble $S$ et al (2017) Influences on the test-retest reliability of functional connectivity MRI and its relationship with behavioral utility. Cereb Cortex 27(11):5415-5429

57. Deco G, Corbetta M (2011) The dynamical balance of the brain at rest Neuroscientist 17(1):107-123

58. Damoiseaux JS, Greicius MD (2009) Greater than the sum of its parts: a review of studies combining structural connectivity and resting-state functional connectivity. Brain Struct Funct 213(6):525-533

59. Friston KJ (2011) Functional and effective connectivity: a review. Brain Connectivity 1(1):13-36

\section{Publisher's Note}

Springer Nature remains neutral with regard to jurisdictional claims in published maps and institutional affiliations.

\section{Submit your manuscript to a SpringerOpen ${ }^{\circ}$ journal and benefit from:}

- Convenient online submission

- Rigorous peer review

- Open access: articles freely available online

- High visibility within the field

Retaining the copyright to your article

Submit your next manuscript at springeropen.com 